\title{
Probing the Structural and Binding Mechanism Heterogeneity of Molecularly Imprinted Polymers
}

\author{
Michael Schauperl ${ }^{\dagger}$ and Dewi W. Lewis* \\ Department of Chemistry, University College London, 20 Gordon St, London WC1H oAJ, United Kingdom
}

\begin{abstract}
We devise a strategy, using a de novo building approach, to construct model molecularly imprinted polymers (MIPs) and assess their ability at binding various target molecules. Whilst our models successfully reproduce the gross experimental selectivities for two xanthines, or atomistic models reveal in detail the considerable heterogeneity of the structure and binding mechanisms of different imprints within such a material. We also demonstrate how nonimprinted regions of a MIP are also responsible for much of binding of target molecules. High levels of crosslinking are shown to produce less specific imprints.
\end{abstract}




\section{Introduction}

Interest in molecularly imprinted polymers (MIPs) ${ }^{1}$ arises from a need for materials which can bind, separate, detect or deliver specific molecules. MIPs are polymers formed in the presence of a "template": the molecule to be subsequently bound or a mimic of that molecule. The template can be bound either non-covalently or covalently, with the former being preferred, as after polymerization the template is more easily removed and the recognition event is similar to the initial templating. The resulting polymer is then said to be imprinted for that template (or a close analogue), having binding sites or cavities which are complimentary in shape and chemistry to the target. Such materials can be used to bind and detect not only small molecules (e.g. altenolol ${ }^{2}$ and Z-resveratrol ${ }^{3}$ ) but also proteins. ${ }^{1 e}$ Promising applications for MIPs include chemo-sensors for blood screening; detection of explosives or chemical and biological weapons; ${ }^{4}$ purification of natural products; ${ }^{5}$ catalysis; ${ }^{6}$ and as slow-release drug delivery systems. ${ }^{7}$

It is sometimes claimed that such materials can, or at least have the potential to, exhibit almost enzyme-like selectivity, being able to distinguish very similar species. But our inability to control individual polymerization steps and determine a detailed structural view of an imprinted polymer makes any control or unambiguous demonstration of such selectivity very difficult.

In contrast to biological macromolecules such as enzymes, which form well-defined three dimensional structures, the structure of MIPs result from a random sequence of monomers and crosslinkers leading to a more disordered structure. ${ }^{8}$ Thus, MIPs contain a structurally heterogeneous distribution of binding sites, likely to have different binding affinities. Moreover, it has been established that only a minority of these sites can be considered truly selective and that the majority of the polymer structure is not imprinted and forms no selective recognition sites. ${ }^{9}$ Similarly, interactions between a template and the growing polymer during synthesis do not necessarily result in the formation of an imprint. Hence, by virtue of the monomer being selected for chemical complementarity with the template, a significant number of non-specific binding sites exist throughout the polymer. It should also be noted that many MIP systems require considerable cross-linking to ensure structural integrity and compatibility with the (typically) liquid phases which they are in contact with, and to provide a rigidity to "lock-in" the imprint.

In separation applications a difference in the average binding of the various analytes may prove sufficient. ${ }^{10}$ However, if specific detection or removal of a target is required, particularly in low concentration or in the presence of other similar species (including a solvent), a significant difference in affinity may be needed. Such a scenario can only be accomplished by the formation of a more homogenous distribution of specific binding site together with a decrease in the number (and affinity) of non-specific binding sites.

But the very nature of amorphous polymers makes determining their molecular structure and physical properties extremely challenging. The performance of a MIP in successfully binding the target is enough to demonstrate that imprinting is successful, but does not reveal any information of the nature of the interactions - they are merely inferred. NMR can determine the relative binding strengths of the functional monomers and the templates but few studies ${ }^{11}$ have considered these interactions with the MIP, and again specific structural models of binding sites are difficult to establish.

Computational studies to date (as summarized by Nicholls et $\mathrm{al}^{12}$ ) have concentrated on the selection of monomers by determining the binding of target molecules and individual (or groups) of monomers. More advanced are Monte Carlo simulations of prepolymerization ensembles containing template, monomers cross-linkers and, solvent. ${ }^{13}$ Most recently, Nicholls and co-workers have explored how prepolymerization mixtures form complexes with the template using molecular dynamics. ${ }^{14}$ Monti et $\mathrm{al}^{15}$ and $\mathrm{Lv}$ et $\mathrm{al}^{16}$ also investigated how single short polymer chains formed interactions with templates. Statistical studies have also been executed where the energy binding site distribution was investigated with a two dimensional lattice. ${ }^{9}$ A recent study used coarse-grained simulations to investigate the binding site distribution of MIPs. ${ }^{17}$ However, we have not found any consideration of assembled MIPs with analyte molecules on an atomistic scale or any analysis of the heterogeneity of MIP cavities or an evaluation of the "goodness of fit" of any cavity for a target molecule.

Whilst there is wide range of published applications ${ }^{\text {tb-g }}$ and a general acceptance of the limitations of such materials due to their amorphous nature, it remains that the molecular recognition mechanisms and capabilities of MIPs are far from being well characterized. More provocatively, perhaps, we can pose the question "how good can a MIP ever be" and is a "perfect" molecular imprint possible? Here, we describe an attempt at using de novo molecular design methods to form realistic MIP networks which are then characterized for selectivity towards their targets (and non-targets). We present examples of where both optimal and non-optimal cavities are formed and their performance compared.

We use the selective binding of caffeine and theophylline by a polymethylacrylic acid polymer, formed from methylacrylic acid (MAA) which is crosslinked using ethylene glycol dimethylacrylate (EGDMA) (scheme 1), as an exemplar of both a useful material ${ }^{18}$ and also one which presents a difficult molecular recognition problem: the two molecules differing only by a methyl substituent.

Our hypothesis is that by "growing" polymers in the presence of the template, optimizing the interactions between the polymer and the template as the polymer is assembled, we can obtain imprints of varying specificity. Moreover, by ensuring the interactions are optimal we can build a model MIP which has the "perfect imprint". Thus, by then characterizing such a polymer, we can determine what would be the best possible performance of a MIP for a series of targets. Indeed, such an approach, where kinetics, solvent and other additional interactions are ignored, rather than being a disadvantage, allows us to amplify the imprinting effect and hence determine a "perfect world" performance.

We take as our starting point the de novo molecular design code ZEBEDDE which, inspired by such approaches for drug design, was initially developed to 
design templates for crystalline porous solids such as zeolites. ${ }^{19}$ The code is able to grow in an evolutionary manner (in the sense that a property, e.g. internal energy, of the growing molecule "improves" with each generation) a molecule from a library of fragments which binds strongly within a target cavity. Here we have the opposite: growing the cavity around the template. ZEBEDDE has been modified for building polymers, with additional control, such as specifying head-to-tail assembly. ${ }^{20}$ More typical polymer building approaches ${ }^{21}$ often require extensive post-construction processing to reproduce experimental densities and structure, frequently requiring non-physical pressure swings and high temperature molecular dynamics.<smiles>Cn1c(=O)c2c(ncn2C)n(C)c1=O</smiles>

(c)<smiles>CC(=O)CCCCn1c(=O)c2c(ncn2C)n(C)c1=O</smiles>

(d)<smiles>CCC(C)(C)C(=O)O</smiles><smiles>CCC(C)(C)C(=O)OCCOC(=O)C(C)(C)CC</smiles>

SCHEME 1. Xanthine templates and analytes (a) caffeine, (b) theophylline and (c) pentoxyphylline used in this work in polymers comprised of (d) methylacrylic acid (MAA) monomers and (e) ethylene glycol dimethylacrylate (EGDMA). The $\mathbf{H}_{\mathrm{A}}$ and $H_{B}$ notation is explained below.

\section{Methods}

The simulations begin with an empty periodic box, in which the polymer will grow. In the case of a MIP, one or more of the template molecules are then randomly placed within this box, whilst for a non-imprinted polymer (NIP) no templates are added. The polymer grows through a series of randomly chosen actions, each of which can be assigned a probability. Beginning from monomers placed within the box, additions to the growing polymer are selected at random from a library consisting of the monomer and the crosslinker, with composition being controlled by the relative probability of selection. New bonds are formed between the selected fragment and polymer as follows. Each monomer has two active hydrogens whilst each crosslinker at least three active hydrogens which can be considered "reactive" (see scheme 2). The reactive hydrogens are labeled either as $\mathrm{H}_{\mathrm{A}}$ or $\mathrm{H}_{\mathrm{B}}$ and only a carbon bonded to $\mathrm{H}_{\mathrm{A}}$ can form a bond with a carbon bonded to $\mathrm{H}_{\mathrm{B}}$, allowing us to build (here) head-to-tail type polymers. ${ }^{20}$ Note that this is not an attempt to mimic in anyway the mechanism of the synthesis: simply a means to build chemically acceptable structure. Torsional rotation can be applied to any bonds formed. Similarly, the entire polymer chain can be displaced or rotated. Additional actions also allow the system to be energy minimized using a molecular mechanics forcefield. ${ }^{22}$ With a user prescribed frequency (thus many actions may be performed before a test is undertaken), actions are accepted if they result in a lowering of the energy of the entire system - otherwise they are rejected. Thus, the system "evolves" along a low potential energy surface: the aim being to optimize local polymer-imprint interactions. The template molecules are considered rigid during the formation of the polymer.<smiles>CCC(C)(CC(C)(C)C(=O)O)C(=O)O</smiles>

Scheme 2. $H_{A}$ and $H_{B}$ are "reactive" sites. A polymer grows when two such hydrogens are eliminated forming a new $\mathrm{C}-\mathrm{C}$ bond

Note that crosslinking of different regions of the same chain or another chain is not currently modeled but, rather, is mimicked through the branching of single chains. Whilst not leading to as rigid a system as in a real MIP, this approach extends beyond previous work. ${ }^{15-16,} 23$ Moreover, we believe that the imprints made in such a branched polymer are more optimal (as they are less rigid) and potentially more typical of imprints on external or macroporous regions of a polymer, where binding of analytes is more likely.

\section{Strategy and Methodology}

The phase space that can be explored in synthesizing a successful MIP is large, particularly the choice of template, monomer and crosslinker; the relative amounts of the reagents; let alone reaction conditions. However, there is no obvious route to the "best" MIP. ${ }^{1 e}$

Clearly the correct choice of functional monomer is paramount as it is the intermolecular interactions with the template and target molecules that give the material its selectivity. NMR binding (titration) studies can aide in the determination of monomer-template binding ${ }^{11}$ and computational studies have also been shown to be useful in this regard. ${ }^{24}$ The ratio of monomer to crosslinker determines the rigidity of the polymer. A more rigid polymer is preferred for practical purposes, being less able to re-arrange and so the imprint "survives". But bulky cross-linkers reduce the closeness of fit (geometrically) between polymer and template.

We now outline our two strategies designed to obtain both general MIP structure and single imprint models.

It should be clear that our building methodology, when multiple templates are considered, will result in a model with a range of imprints (of varying specificity) together with many non-specific sites. Such models are 
useful in obtaining a general understanding of the homogeneity of the binding sites. A second strategy is to build models of single binding sites around one template in such a way as to allow the polymer to optimize to give the "best" imprint. We can then generate an ensemble of such single imprint models and again consider their heterogeneity, but also now be able to focus on how they interact with the target analytes, gaining local structural knowledge of what makes a "good" and "bad" imprint.

For the former strategy we took a cubic box of side $50 \AA$ with seven template molecules randomly placed for the MIP systems and an empty box for the non-imprinted polymer (NIP). The simulation was continued until a density of $0.65 \mathrm{~g} \mathrm{~cm}^{-3}$ was reached, which based on preliminary simulations was the maximum achievable in a reasonable simulation time. Further densification is difficult with new additions requiring considerable perturbation of the existing structure; which we wish to avoid as the aim is to build optimized imprints. The experimental bulk density of MAA/EDGMA polymers is ca. $1.05 \mathrm{~g} \mathrm{~cm}^{-3} .{ }^{25}$ However, we believe that the structures we have formed are representative of external and macropore surfaces of the polymer and hence appropriate models to consider how analytes may be absorbed. This assumption is based on evidence that the density in the surface region is lower than in the bulk of the polymer. ${ }^{26}$ We can also expect that the surface imprints will be those more readily accessed due to slower diffusion into the bulk and hence reproducing the experimental bulk density may not be particularly relevant. Representative snapshots during such a simulation are given in Figure 1.
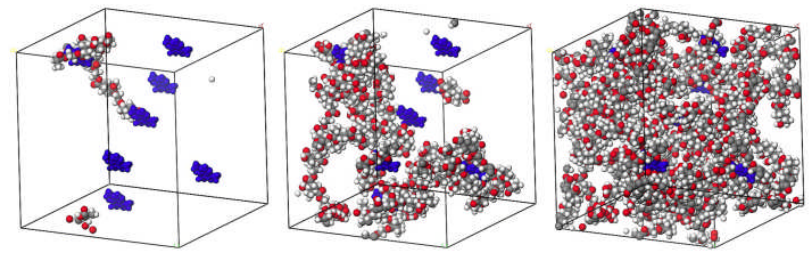

Figure 1. Snapshots from a ZEBEDDE run where multiple polymer chains were built in the presence of multiple template molecules.

For the second approach we used a smaller $20 \AA$ cubic box with a single template. Building was terminated when additions to the growing chain failed for 1000 attempts.

At the end of the building process, each system was energy minimized with the template in place. The Discover $\operatorname{code}^{27}$ was used throughout with the pcff forcefield. ${ }^{22}$ The electrostatics were considered via the particle mesh Ewald method and all real-space (shortrange) cut-offs used being $9.5 \AA$ with a smooth spline of width $1 \AA$. In later simulations we have also performed molecular dynamics simulations (using the same code and forcefield) prior to minimization. Each of these approaches is used to ensure optimal host-guest interaction, since ZEBEDDE actions are limited to local optimization of inter- and intramolecular interactions. In certain cases the MIP has been optimized in the absence of the template in order to mimic the experimental system more accurately.
Canonical Monte Carlo (CMC) simulations were performed using the Sorption package in Materials Studio ${ }^{27}$ to identify and characterize the various imprints and more general non-specific binding sites. Note we do not consider here any entropic contribution which will be significant - future work will consider the impact of solvation and temperature on these imprints.

The notation MIP-template is used to denote a polymer that has been formed in the presence of the stated template with the abbreviation THEO, CAF and PENT. Thus a polymer formed in the presence of caffeine is denoted MIP-CAF.

\section{Results and Discussion}

We exemplify first (Supplementary Information Figure 1) that our method is capable of forming appropriately structured polymers. ZEBEDDE is able to reproduce both amorphous and crystalline regions in syndiotactic polystyrene, the 3 -fold screw axis ${ }^{28}$ clearly being reproduced. Amorphous dichloroxylene systems with high porosity were also previously successfully modelled. ${ }^{20}$

Our efforts here are primarily to establish that our modeling approach is suitable and to attempt to firmly determine the mode of action of MIPs in general. Thus it is worthwhile summarizing what is established experimentally for our test system. According to Wang et $a l .{ }^{18}$ a theophylline imprinted MIP binds theophylline in preference to caffeine. However, a caffeine imprinted MIP preferentially bound theophylline, although it does also successfully bind caffeine. Furthermore, both imprinted MIPs showed enhanced uptakes of both molecules compared to a NIP. We note further that these observations concur with the arguments of Dauwe and Sellergren $^{29}$ who suggested that the best recognition behavior should be found for a MIP templated with theophylline. They showed that a higher Brønsted basicity leads to a higher recognition factor when a monomer with an acid function is used, whilst the hydrophobicity of the template molecule has little influence on the interactions with the polymer.

We begin by considering the general binding behavior of the MIP systems formed in the presence of multiple templates. A ratio of 1:1 between monomer and crosslinker were applied. MIP assemblies imprinted with each of the three templates, together with a NIP, were energy minimized with the template in place. The templates were removed and CMC simulations were then performed with caffeine and theophylline as sorbents at a fixed loading of 10 sorbent molecules in the simulation cell (a volume of $125000 \AA^{3}$ ) at a temperature of 298K.

We can see (Figure 2 - (a) and (b)) that theophylline generally binds stronger than caffeine in all cases. This is due to the basic group in theophylline interacting with the acid function of the polymer. Considering the uptake of theophylline we note that the MIP-THEO has resulted in a significant number of theophylline specific imprints (Figure 2(a)) whilst theophylline binds less strongly with the imprints in the MIP-CAF and MIPPENT. The strongly binding polymer-theophylline interaction is emphasized by the fact that the least specific theophylline-polymer interactions in the NIP remain 
stronger than those in the caffeine and pentoxifylline imprinted MIPs. In contrast, the imprinting effects seem less evident for caffeine with little difference in binding energy between the three MIPs formed. Moreover, since the binding of caffeine by the NIP is comparable to the MIPs, there appears little evidence of any shape selective sites capable of enhanced binding, implying that unless there are specific strong interactions between the polymer and the sorbate, we cannot expect any selectivity for caffeine.

We performed MD simulations (NVT ensembles at 298 $\mathrm{K}$ for $5 \mathrm{ps}$ with a timestep of $1 \mathrm{fs}$, all atoms were free to move) of MIP-CAF, MIP-THEO (and for comparison the NIP) with the template in place with the expectation that the imprints would be optimized and result in enhanced binding and selectivities. However, this proved not to be in evidence (Figure 2 (c)-(d)). MIPCAF now has some stronger binding sites than prior to optimization, whilst in the case of theophylline, we have lost some of the strongest binding sites. We also see that the general profile for MIP-CAF is now very similar to the as-formed NIP system. A number of theophylline specific sites in MIP-CAF remain, but with a lower binding energy than prior to optimization. For caffeine the behavior is similar to the non-optimized systems, with the NIP and MIP-THEO being the more strongly interacting, which would again suggest that the caffeine is not binding in any specific manner to the imprints but rather with the non-imprinted regions of the polymer.
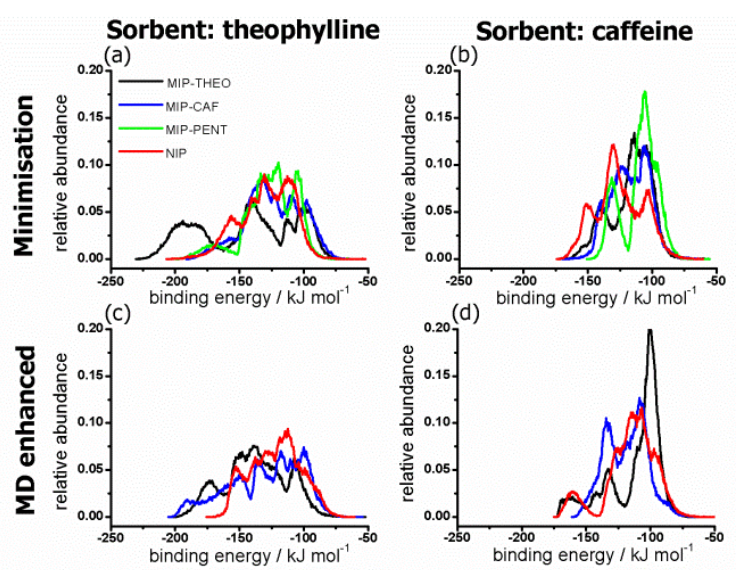

Figure 2: Binding energies of xanthines obtained from CMC simulations for NIP and MIPs.

We can explain this loss of imprinted behavior as simply that the non-imprinted regions of the polymer can also relax, leading to an overall "un-optimization" of the imprint region as the overall simulation cell minimizes its energy. After all, these polymers typically consist of 250 monomers and cross-linkers but only 7 template molecules. Experimentally monomer to template ratios are often 1:1 and we might expect such behavior to be therefore lessened than we observe.

Thus, based on the above simulations, we conclude that the MIPs are certainly exhibiting some specific interactions with the templates, behaving as observed experimentally, with theophylline binding more strongly than caffeine to any MIP or NIP and that the most selective system being MIP-THEO. Moreover, our models have captured the general heterogeneity of MIP (and NIP) binding sites. We may also conclude that to ensure specificity, the number density of imprints must be maximized as otherwise saturation effects play an important role; ${ }^{30}$ by increasing the relative concentration of the template in the synthesis. However, this will compromise the specificity of each imprint as the polymer will not be able to form "high quality" imprints around each template: we will see below how a single imprint in a smaller box does not give higher binding.

The canonical MC approach probes the average performance of the polymer system. We now focus on the binding specifically within the imprints that formed around each template, to identify the host-guest chemistry occurring. For the caffeine and theophylline imprinted polymers we performed energy minimization and MD simulations, first with the original template in place and then by replacing the template with the other xanthine (i.e. we convert caffeine to theophylline in silico and vice versa). We thus gain an average binding energy (in eight simulated MIPs for each system) for each of the imprints. For the NIP we place the xanthine at sites identified as the most strongly binding in the CMC calculations (above).

\begin{tabular}{|l|l|l|l|}
\hline \multicolumn{4}{|c|}{ Relative Binding Energy ${ }^{*} / \mathrm{kJ} \mathrm{mol}^{-1}$} \\
\hline \multicolumn{4}{|c|}{ MIP Minimization } \\
\hline & MIP-THEO & MIP-CAF & NIP \\
\hline Theophylline & -64 & -34 & -19 \\
\hline Caffeine & -49 & -42 & 0 \\
\hline MIP MD and minimization \\
\hline \multicolumn{4}{|l|}{} \\
\hline Theophylline & -73 & -38 & -24 \\
\hline Caffeine & -46 & -48 & -21 \\
\hline
\end{tabular}

Table 1. Relative binding energies of the xanthines in both MIP and NIP environments.

*Binding energy is defined as the difference in between the minimized energy of the polymer and analyte (isolated gas phase) and that of the bound polymer-analyte system. Values are given relative to the least well bound (caffeine in the NIP).

Gratifyingly, we now see (Table 1 ) that the designing of the imprint for specific templates is successful, with every MIP showing the highest affinity for its template. We see a difference of $c a$. 10-15 $\mathrm{kJ} \mathrm{mol}^{-1}$ between the template and the other xanthine, with the molecular dynamics improving the binding and the selectivity as we first postulated. The stronger binding of theophylline over caffeine in the NIP is as expected, ${ }^{29}$ but is not particularly distinct from the MIP performance for the minimized systems. The selectivity for theophylline is now diminished, presumably as the empty polymer rearranges to maximize inter-chain interactions. However, the selectivity found for MIP-CAF is "better" than the experimental result, ${ }^{18}$ where theophylline is more favorably bound than caffeine. But, note that theophylline is more strongly bound than caffeine in our NIP, as 
in experiment. Therefore, we conclude that the higher observed experimental retention factor for theophylline in a caffeine imprinted $\mathrm{MIP}^{18}$ is maybe more the result of additional non-specific binding of the amino group and the acidic function of the polymer and not any limitation of the actual imprinted region. Ideally therefore, increasing the proportion of imprints versus nonimprinted regions will improve selectivity for caffeine, all other factors being equal. In the context of our simulations, these results validate our approach in being able to design very efficient imprints and that they represent the more specific sites present (amongst many others) in a real polymer. Note that we do not consider the differences between the binding of caffeine in the different MIPs to be significant as there are fewer specific intermolecular interactions, as we discuss below.

We now focus on the single imprints formed using our second strategy. This approach allows us to build a number of optimal imprints formed in the same volume, with minimal NIP character. We considered monomer to crosslinker ratios of 2:3 and 1:20. For each composition we performed twenty simulations, which gave polymers ranging in density from 0.5 to $1.1 \mathrm{~g} \mathrm{~mol}^{-1}$, which now mimic both surface and macroporous regions (as before) and the more dense bulk regions (experimentally ${ }^{25} \mathrm{ca} .1 .05 \mathrm{~g} \mathrm{~mol}^{-1}$ ).

Every imprint formed is different, as in a real polymer, so it is not easy to describe their structure in a general way. However, what will become clear is that imprints are heterogeneous in their selectivity for caffeine and theophylline, but that in general each imprint is marginally more selective for its template. Hence, we report the relative affinity for both: each MIP is energy minimized with the original template and then with the competing xanthine. Plotting the binding energies obtained against each other now allows us to compare the binding strength and selectivity at the same time (Figure 3 ).

What is immediately apparent is that, even though each simulation is controlled by the same input (box size etc.) and has evolved to optimize the polymer-template interactions, there remains a large distribution of binding strengths. Moreover, the majority show little selectivity (they are very close to the 1:1 line). We see some selectivity of the MIP-CAF towards the caffeine sorbent, but the MIP-THEO shows no selectivity for theophylline, in contrast to the larger systems above, where there are more non-specific sites.

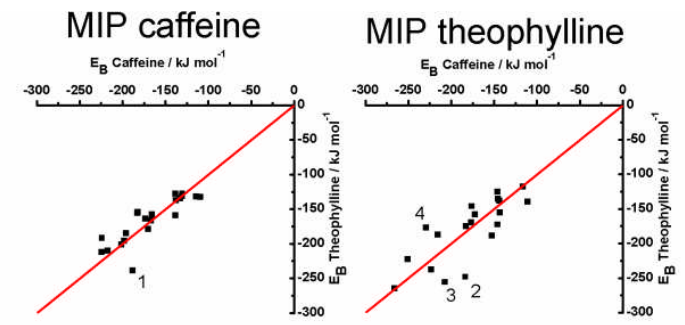

FIGURE 3. Binding energies of caffeine and theophylline in MIP-CAF and MIP-THEO. Thus if both xanthines bind equally in a MIP, they lie on the 1:1 line plotted.
The results here are perhaps disappointing, revealing little selectivity, compared to the CMC results. However, we believe this is mainly because we have made denser materials in these simulations, which may not be representative of neither the imprinted regions nor those actually accessed in a real material. Consider how these imprints form. The polymer chain evolves to maximize the interactions between template and polymer. Therefore, we might expect more dense materials to have more of the polymer in close proximity to the template. But we also optimize the structure of the polymer which - due to the high density - is strained and so the imprints become less well defined on relaxation of the entire simulation box. Conversely in our less dense ensembles, relaxation of the system actually enhanced the template-polymer interactions as there was less internal strain on the polymer chain. Thus, we may conclude that whilst obtaining a bulk density comparable to experiment may seem a good outcome, the poor performance of the imprints in these denser systems supports our view that the more valuable imprints are at the surface or in macropores. Whilst such imprints may be less rigid than those in the bulk they are in fact more selective because they can optimize during synthesis. Moreover, these simulations can be thought of as implying that most of the recognition events occur at less dense, surface regions of the polymer, where nonimprinted regions which preferentially bind theophylline, will also be prevalent.

Nevertheless, we have formed a number of imprints which show considerable selectivity for the template around which they formed. But, we also have a few which show similar high selectivity for the other xanthine. We therefore analyze the structure of a number of these imprints to determine how this specificity arises.

The first imprint we consider (marked with $\mathbf{1}$ in Figure 3) was formed in the presence of caffeine but shows high selectivity towards theophylline. We find that there are hydroxyl groups both deep in the cavity and at its edge (Figure 4). Thus caffeine, whose methyl group prevents the molecule fully entering the cavity, binds through the hydroxyl groups at the edge of the imprint. In contrast, theophylline can bind with this deeper hydroxyl, in addition to those further out, forming an additional hydrogen bond thus being more strongly bound than caffeine. Thus, we can see that what was a poor interaction between the functional monomer and the caffeine during the formation of the imprint provides an additional strong binding site for theophylline. Thus, whilst the shape of the imprint is correct for caffeine, the orientation of the functional groups in theophylline lead to a more strongly bound molecule. These types of imprints may contribute to the experimental observation that theophylline is more strongly bound than caffeine in caffeine-imprinted MIPs, ${ }^{18}$ together with the fact that non-imprinted regions will also generally bind more strongly to theophylline. ${ }^{29}$ Such general polymer-analyte interactions are a by-product of the large number of possible interactions present in systems where the functional monomer is in excess, and could be minimized if alternative, non-functional monomers were also present (a co-polymer). 


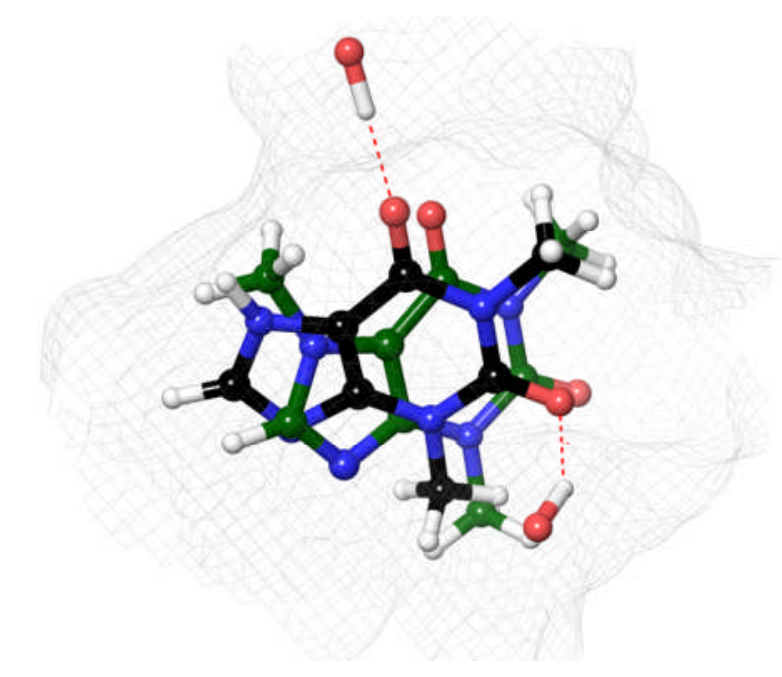

Figure 4. Structure of an imprint formed with caffeine but which shows higher selectivity for theophylline. The theophylline can locate deeper in the imprint allowing the formation of an additional hydrogen bond.

We now consider an imprint which selective binds its template (marked 2 in Figure 3). The imprint shown in Figure 5 was imprinted for theophylline and selectively binds it over caffeine. Inspection of the structure shows us that the imprint is narrow in the region of the nitrogen $\left(\mathrm{N}_{7}\right)$ of the theophylline and thus a caffeine molecule, which has the methyl group attached at this position, cannot geometrically fit in the same location in the imprint. We can demonstrate this by simply adding a methyl group to the theophylline (in situ) and reminimizing. We find that the caffeine is ejected from the initial bound position, with the imprint rearranging significantly, and whilst the caffeine still interacts with the hydroxyl groups on the outer part of the imprint, it is less well bound than the theophylline. We can consider the imprint to be "steric only" with no specific intermolecular interactions for caffeine.

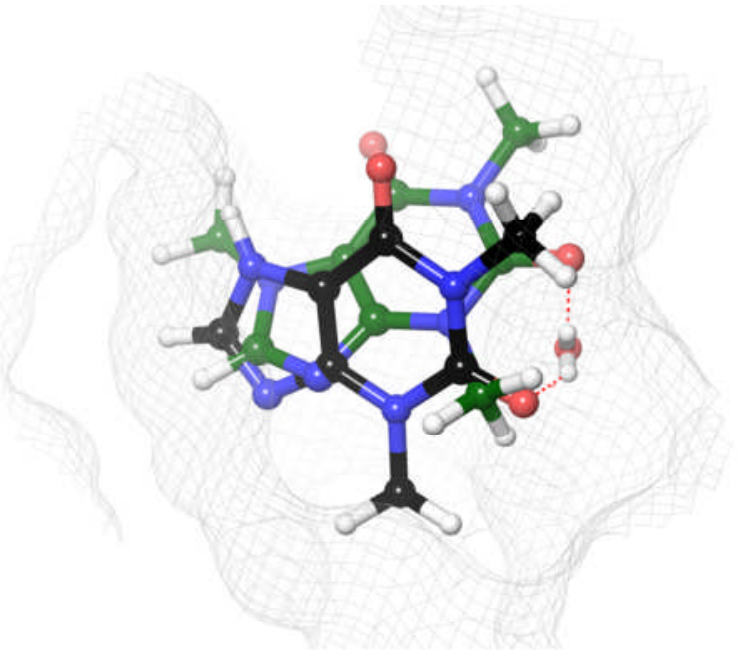

Figure 5: Imprint in MIP-THEO which is selective for theophylline. The methyl group of caffeine (lighter molecule carbon colored green) prevents it from fitting the imprint as well as theophylline (darker molecule - carbon colored grey) even though it can also bind to the hydroxyl group of the imprint.
Whilst the imprint above relies on the shape difference between caffeine and theophylline, others are present which show enhanced selectivity due to the attractive intermolecular interactions. Figure 6 shows an imprint (marked 3 in Figure 3) in which both xanthines occupy the imprint with very similar geometries. But we find that this imprint has an enhanced selectivity for theophylline (around which it formed) due to the hydrogen bond between the polymer and the hydrogen acceptor group $\mathrm{N}_{5}$ of the theophylline and a further weak hydrogen bond between the hydrogen on $\mathrm{N}_{7}$ and oxygen in the polymer. The latter is not present in caffeine and instead we have a repulsive contribution due to the methyl group. Thus the selective binding here is due to the attractive intermolecular interactions that were formed during the formation of the polymer in the simulation: what we might refer to as the preferred type of imprint.

The final imprint we consider is one imprinted with theophylline but preferentially binds caffeine (marked 4 in Figure 3). In contrast to the first imprint considered (Figure 4), the imprinted position of theophylline is not optimal. Moreover, when theophylline is changed to caffeine, there is sufficient room in the imprint for the caffeine to re-orient significantly (Figure 7) and find a sterically more favorable site. This now optimal site for caffeine will also bind theophylline (which we do by now removing the methyl group and re-optimizing) with a binding energy similar to caffeine, and now stronger than in the position where is was imprinted. This example demonstrates how non-specific interactions could well dominate a MIP and that even successful recognition events may not be due to the imprints formed for that template.

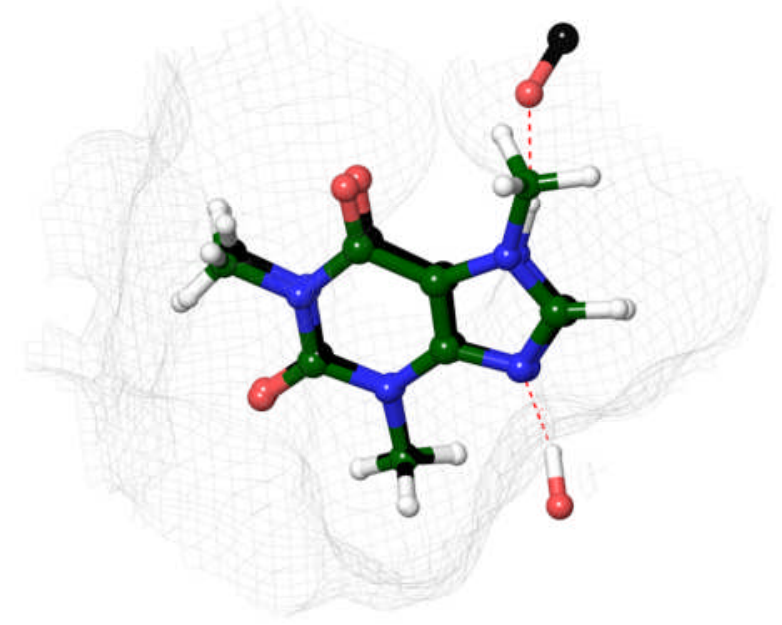

Figure 6: Imprint formed with theophylline, which shows selectivity for theophylline over caffeine. Both molecules occupy essentially the same site but the theophylline can form an additional hydrogen bond. 


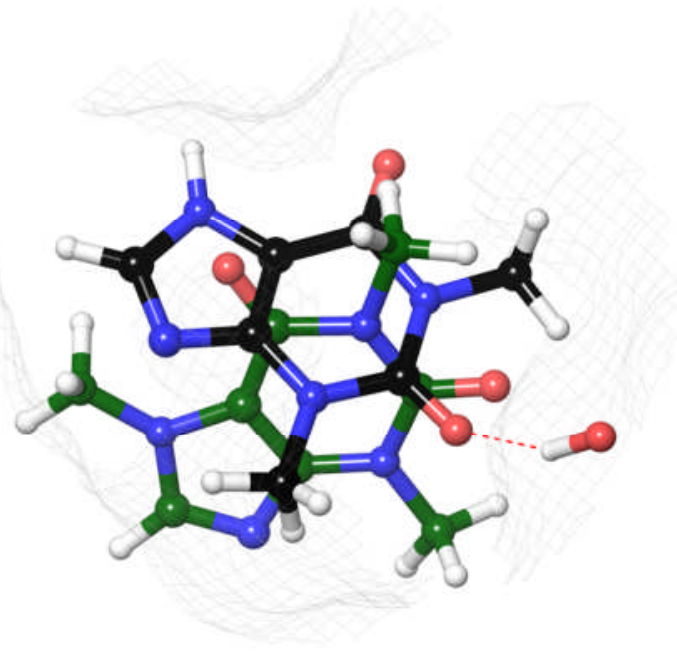

Figure 7. An imprint formed around theophylline but which binds caffeine more strongly due to its shape being more complimentary and the binding site for theophylline being non-optimal, with only one hydrogen bond formed.

It is impractical to continue to describe every imprint. However, generally, we have found that each strongly binding imprint has formed in such a way as to allow the formation of hydrogen bonds between both ketone groups of the xanthine and the $\mathrm{OH}$ groups of the functional monomer. Conversely, less strongly binding imprints tends to be more open in their structure.

We now consider how the imprint might change on exposure to analytes by studying the dynamics of the polymer with the template and then the competitor in the imprint. We took the minimized MIPs (the same ensemble discussed in Figure 3) and performed molecular dynamics simulations (NVT ensembles at $298 \mathrm{~K}$ for 5 ps with a timestep of $1 \mathrm{fs}$ ) with the xanthines present. The MD step allows the optimization of the xanthineMIP interactions and so the binding of the xanthine with the MIP is enhanced regardless of which xanthine was present initially (Figure 8): in the majority of cases, the binding post-MD is stronger than the initial energy minimized only case. Thus we conclude that the addition of an MD step will result in more realistic and optimal model imprint from a modelling perspective. However, these results may suggest that imprints in a real polymer also change over time. For example, the binding strength of both caffeine and theophylline increase in the MIP-CAF with the MD (Figure 9(c-d)), which suggests that imprints exposed to mixtures may change specificity over time.

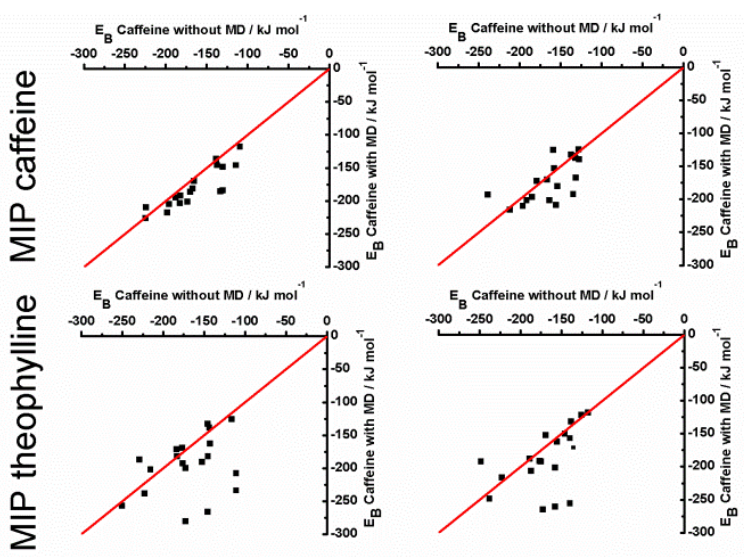

Figure 8. Binding energies of the analyte before and after performing an MD simulation with the analyte in the imprint. The top two graphs representing the change of energy in MIP-CAF with caffeine (left) and theophylline (right). Similarly, the bottom graphs are for MIP-THEO.

Finally, we investigate the relationship between the extent of cross-linking and the binding energy of the imprints. The presence of the large rigid crosslinker will inhibit the formation of small imprints that closely follow the geometry of the templates. Recall also, as we have seen above, that the presence of too many functional groups that can bind to target molecules in an imprint may lead to unforeseen binding of competitor molecules, which will be mitigated by the presence of crosslinker.

We constructed a series of single imprint MIPs with a range of monomer:crosslinker ratios. From Figure 9 we see that imprints made with higher monomer concentrations do generally bind more strongly, as more functional groups able to form strong interactions are close to the template. However, we also see, by simply considering the dispersion of binding energies found compared to the non-crosslinked systems (Figure 3), that changes in crosslinker concentration leads to imprints that are more heterogeneous in their affinity, more so than between the different imprints formed at lower crosslinker concentrations. We conclude therefore that, whilst it is generally better to have imprints with higher monomer content to give strongly binding imprints, it gives us no certainty that the imprint will be optimal solely because of this, as the monomers can be in the wrong position/orientation. Moreover, higher crosslinker concentration leads to an increase in the heterogeneity of the imprints formed. But there will also be a reduction in the amount of non-imprinted polymer backbone to bind with any analyte (as in a NIP), which can of course make a MIP appear selective. Whilst we have considered over 80 different imprints here, we have not considered (as we did previously) the heterogeneity of binding at a single crosslinker concentration. Therefore, we cannot state at present if these findings are necessarily statistically significant. Further work will be undertaken to probe this result further and the impact of the presence of cross-linker on the structural integrity of the imprints as we have already shown how they are modified in the non-crosslinked case. 

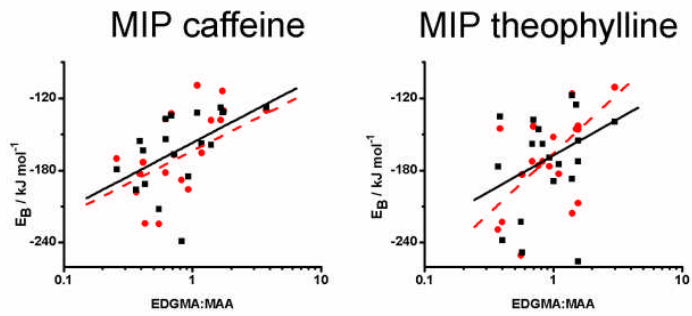

Figure 9: Binding energy as function of the crosslinker concentration (caffeine: circles; theophylline: squares). Lines represent least square fit (caffeine dashed line).

\section{Conclusions}

The novel strategy developed here is shown to produce models of MIPs consistent with the generally accepted understanding of MIP action. Furthermore, the model imprints generated correlate well with experimental data $^{18}$ on the detection of caffeine and theophylline.

Our calculations clearly show that imprinted regions do indeed preferentially bind the template over the competing molecule xanthine. But we also observe different types of imprints: ones where the shape determines the specificity and others where specific intermolecular interactions dominate.

For the particular system considered here, where the two analytes are very similar in structure, it appears that imprinted regions are most effective for the analyte (theophylline) with the maximal intermolecular interaction with the polymer. However, much of the specific binding of the most favored species remains due to interaction with non-imprinted regions of the polymer. Whilst therefore it would appear that increasing the number of possible imprints may lead to improved selectivity, there is evidence here that such an approach will fail as the intramolecular forces within the polymer strands will dominate during the synthesis, resulting in the formation of more less-specific imprints.

Our consideration of cross-linking suggests that a route to more homogenous imprints and fewer non-specific sites during synthesis would be possible if we could ensure a high concentration of monomer in the vicinity of the template with the other regions having a higher concentration of crosslinker. Thus, if experimentally possible, low temperature aging of the template with small polymer oligomers prior to cross-linking may be a possible route to enhanced performance. Similarly it may be profitably to consider larger monomers comprising functional and non-functional regions, to minimize the portions of the polymer which bind analytes in non-imprinted regions. These results also demonstrate that whilst bulky crosslinkers impart structural integrity, they also inhibit the formation of optimal imprints. Thus, alternative strategies for crosslinking may be preferred.

We believe our approach is capable of providing new insight into the molecular action of MIPs in general, insight difficult to obtain from experiment. Our ap- proach neglects any consideration of many experimental parameters, focusing entirely on ensuring that the polymer built and the interactions with the template are optimal: in essence we are building idealized MIPs. But by doing so, we determine the best possible performance of an imprint and also of the nonimprinted regions for the interaction with analytes. These structural models complement the approaches of others $^{12,14}$ in considering pre-polymerization mixtures. Our approach allows us to determine the heterogeneity of imprints in a polymer system and hence to identify preferred experimental conditions which will generate MIPs with improved performance. Enhancements to our models through MD annealing, more realistic crosslinking strategies and the inclusion of solvation will be the focus of further work. Similarly we can consider how template-template interactions influence the recognition event and imprint formation, as highlighted recently by simulations by Olsson et $\mathrm{al}^{14}$ together with the influence of entropic contributions as illustrated by Dourado et al. ${ }^{31}$

The simulation strategy developed here now allows us to consider further systems and explore in more detail the performance of such materials in a more critical manner. However, our results also demonstrate how the very nature of these materials may inhibit the development of highly selective MIPs.

\section{Supporting Information}

Structure of isotactic polystyrene to illustrate building process is included in Supporting Information. This material is available free of charge via the Internet at http://pubs.acs.org.

\section{AUTHOR INFORMATION}

\section{Corresponding Author}

Dewi W. Lewis. d.w.lewis@ucl.ac.uk

\section{Present Addresses}

† Institute of General, Inorganic and Theoretical Chemistry, University of Innsbruck, Innrain 8o-82, 6020 Innsbruck, Austria

\section{Author Contributions}

Both authors contributed equally.

\section{Notes}

The authors declare no competing financial interests.

\section{REFERENCES}

1. (a) Whitcombe, M. J.; Kirsch, N.; Nicholls, I. A., Molecular Imprinting Science and Technology: A Survey of the Literature for the Years 2004-2011. J. Mol. Recognit. 2014, 27, 297401; (b) Haupt, K.; Mosbach, K., Molecularly Imprinted Polymers and Their Use in Biomimetic Sensors. Chem. Rev. 2ooo, 100, 24952504; (c) Bossi, A.; Bonini, F.; Turner, A. P. F.; Piletsky, S. A., Molecularly Imprinted Polymers for the Recognition of Proteins: The State of the Art. Biosens. Bioelectron. 2007, 22, 1131-1137; (d) Motherwell, W. B.; Bingham, M. J.; Six, Y., Recent Progress in the Design and Synthesis of Artificial Enzymes. Tetrahedron 20o1, 57, 4663-4686; (e) Whitcombe, M. J.; Chianella, I.; Larcombe, L.; Piletsky, S. A.; Noble, J.; Porter, R.; Horgan, A., The Rational Development of Molecularly Imprinted Polymer-Based Sensors for Protein Detection. Chem. Soc. Rev. 2011, 40, 1547-1571; (f) Sellergren, B., Imprinted Polymers with Memory for Small Molecules, Proteins, or Crystals. Angew. Chem. Int. Ed. 20oo, 39, 
1031-1037; (g) Sellergren, B., Noncovalent Molecular Imprinting: Antibody-Like Molecular Recognition in Polymeric Network Materials. TrAC, Trends Anal. Chem. 1997, 16, 310-320.

2. Cheong, W. J.; Yang, S. H.; Ali, F., Molecular Imprinted Polymers for Separation Science: A Review of Reviews. J. Sep. Sci. 2013, 36, 609-628.

3. Wang, L.; Zhang, Z., Molecular Imprinted PolymerBased Chemiluminescence Imaging Sensor for the Detection of Trans-Resveratrol. Anal Chim Acta 2007, 592, 115-20.

4. Burnworth, M.; Rowan, S. J.; Weder, C., Fluorescent Sensors for the Detection of Chemical Warfare Agents. Chem. Eur. J. 2007, 13, 7828-7836.

5. Chen, L.; Xu, S.; Li, J., Recent Advances in Molecular Imprinting Technology: Current Status, Challenges and Highlighted Applications. Chem. Soc. Rev. 2011, 40, 2922-2942.

6. Meng, Z.; Yamazaki, T.; Sode, K., A Molecularly Imprinted Catalyst Designed by a Computational Approach in Catalysing a Transesterification Process. Biosens. Bioelectron. 2004, 20, 1068-1075.

7. Cunliffe, D.; Kirby, A.; Alexander, C., Molecularly Imprinted Drug Delivery Systems. Adv. Drug Deliv. Rev 2005, 57, 1836-1853.

8. Muhammad, T.; Nur, Z.; Piletska, E. V.; Yimit, O.; Piletsky, S. A., Rational Design of Molecularly Imprinted Polymer: The Choice of Cross-Linker. Analyst 2012, 137, 2623-2628.

9. Wu, X.; Carroll, W. R.; Shimizu, K. D., Stochastic Lattice Model Simulations of Molecularly Imprinted Polymers. Chem. Mater. 2008, 20, 4335-4346.

10. Ambrosini, S.; Serra, M.; Shinde, S.; Sellergren, B.; De Lorenzi, E., Synthesis and Chromatographic Evaluation of Molecularly Imprinted Polymers Prepared by the Substructure Approach for the Class-Selective Recognition of Glucuronides. J. Chromatogr. A 2011, 1218, 6961-6969.

11. Skogsberg, U.; Meyer, C.; Rehbein, J.; Fischer, G.; Schauff, S.; Welsch, N.; Albert, K.; Hall, A. J.; Sellergren, B., A Solid-State and Suspended-State Magic Angle Spinning Nuclear Magnetic Resonance Spectroscopic Investigation of a 9Ethyladenine Molecularly Imprinted Polymer. PPTEn 20o7, 48, 229-238.

12. Nicholls, I. A.; Andersson, H. S.; Charlton, C.; Henschel, H.; Karlsson, B. C.; Karlsson, J. G.; O’Mahony, J.; Rosengren, A. M.; Rosengren, K. J.; Wikman, S., Theoretical and Computational Strategies for Rational Molecularly Imprinted Polymer Design. Biosens. Bioelectron. 2009, 25, 543-552.

13. Herdes, C.; Sarkisov, L., Computer Simulation of Volatile Organic Compound Adsorption in Atomistic Models of Molecularly Imprinted Polymers. Langmuir 2oo9, 25, 5352-5359.

14. Olsson, G. D.; Karlsson, B. C. G.; Schillinger, E.; Sellergren, B.; Nicholls, I. A., Theoretical Studies of 17-BetaEstradiol-Imprinted Prepolymerization Mixtures: Insights Concerning the Roles of Cross-Linking and Functional Monomers in Template Complexation and Polymerization. Ind. Eng. Chem. Res. 2013, 52, 13965-13970.

15. Monti, S.; Cappelli, C.; Bronco, S.; Giusti, P.; Ciardelli, G., Towards the Design of Highly Selective Recognition Sites into Molecular Imprinting Polymers: A Computational Approach. Biosens. Bioelectron. 2006, 22, 153-163.

16. Lv, Y.; Lin, Z.; Tan, T.; Feng, W.; Qin, P.; Li, C., Application of Molecular Dynamics Modeling for the Prediction of Selective Adsorption Properties of Dimethoate Imprinting Polymer. Sensors and Actuators B: Chemical 2oo8, 133, 15-23.

17. Levi, L.; Raim, V.; Srebnik, S., A Brief Review of CoarseGrained and Other Computational Studies of Molecularly Imprinted Polymers. J. Mol. Recognit. 2011, 24, 883-891.

18. Wang, D.; Hong, S.; Row, K., Solid Extraction of Caffeine and Theophylline from Green Tea by Molecular Imprinted Polymers. Korean J. Chem. Eng. 2004, 21, 853-857.

19. (a) Lewis, D. W.; Willock, D. J.; Catlow, C. R. A.; Thomas, J. M.; Hutchings, G. J., De Novo Design of StructureDirecting Agents for the Synthesis of Microporous Solids. Natur 1996, 382, 604-6o6; (b) Willock, D. J.; Lewis, D. W.; Catlow, C. R. A.; Hutchings, G. J.; Thomas, J. M., Designing Templates for the Synthesis of Microporous Solids Using De Novo Molecular Design Methods. J. Mol. Catal. A: Chem. 1997, 119, 415-424; (c) Lewis, D. W.; Sankar, G.; Wyles, J. K.; Thomas, J. M.; Catlow, C. R. A.; Willock, D. J., Synthesis of a Small-Pore Microporous Material
Using a Computationally Designed Template. Angew. Chem. Int. Ed. 1997, 36, 2675-2677; (d) Sankar, G.; Wyles, J. K.; Jones, R. H.; Thomas, J. M.; Catlow, C. R. A.; Lewis, D. W.; Clegg, W.; Coles, S. J.; Teat, S. J., Structure of Templated Microcrystalline Daf-5 (Coo.28alo.72po4c1oh2on2) Determined by Synchrotron-Based Diffraction Methods. Chem. Commun. 1998, 117-118.

20. Trewin, A.; Willock, D. J.; Cooper, A. I., Atomistic Simulation of Micropore Structure, Surface Area, and Gas Sorption Properties for Amorphous Microporous Polymer Networks. J. Phys. Chem. C 2008, 112, 20549-20559.

21. Theodorou, D. N.; Suter, U. W., Detailed MolecularStructure of a Vinyl Polymer Glass. Macromolecules 1985, 18, 14671478 .

22. (a) Sun, H., Compass: An Ab Initio Force-Field Optimized for Condensed-Phase Applications Overview with Details on Alkane and Benzene Compounds. J. Phys. Chem. B 1998, 102, 7338-7364; (b) Sun, H.; Ren, P.; Fried, J., The Compass Force Field: Parameterization and Validation for Phosphazenes. Comput. Theor. Polym. Sci. 1998, 8, 229-246; (c) Paton, R. S.; Goodman, J. M., Hydrogen Bonding and Pi-Stacking: How Reliable Are Force Fields? A Critical Evaluation of Force Field Descriptions of Nonbonded Interactions. J. Chem. Inf. Model. 2009, 49, 944-55; (d) Maple, J. R.; Hwang, M. J.; Stockfisch, T. P.; Dinur, U.; Waldman, M.; Ewig, C. S.; Hagler, A. T., Derivation of Class Ii Force Fields. I. Methodology and Quantum Force Field for the Alkyl Functional Group and Alkane Molecules. J. Comput. Chem. 1994, 15, 162-182; (e) Rigby, D.; Sun, H.; Eichinger, B., Computer Simulations of Poly (Ethylene Oxide): Force Field, Pvt Diagram and Cyclization Behaviour. Polym. Int. 1997, 44, 311-330.

23. Larsen, G. S.; Lin, P.; Hart, K. E.; Colina, C. M. Molecular Simulations of Pim-1-Like Polymers of Intrinsic Microporosity. Macromolecules 2o11, 44, 6944-6951.

24. Nicholls, I. A.; Andersson, H. S.; Charlton, C.; Henschel H.; Karlsson, B. C. G.; Karlsson, J. G.; O'Mahony, J.; Rosengren, A. M.; Rosengren, K. J.; Wikman, S., Theoretical and Computational Strategies for Rational Molecularly Imprinted Polymer Design. Biosens. Bioelectron. 2009, 25, 543-552.

25. Anderson, H. H., Triisopropylgermanium Esters. J. Org. Chem. 1955, 20, 536-541.

26. (a) Forster, S.; Wenz, E.; Lindner, P., Density Profile of Spherical Polymer Brushes. Phys. Rev. Lett. 1996, 77, 95-98; (b) Milner, S. T.; Witten, T. A.; Cates, M. E., A Parabolic Density Profile for Grafted Polymers. Europhys. Lett. 1988, 5, 413-418.

27. Materials Studio. Accelrys Software Inc., S. D., Discovery Studio Modeling Environment, Release 4.o. 2013 Available from http://accelrys.com/products/materials-studio/. 28. Natta, G.; Corradini, P.; Bassi, I., Crystal Structure of Isotactic Polystyrene. Il Nuovo Cimento (1955-1965) 196o, 15, 68-82. 29. Dauwe, C.; Sellergren, B., Influence of Template Basicity and Hydrophobicity on the Molecular Recognition Properties of Molecularly Imprinted Polymers. J. Chromatogr. A 1996, 753, 191200 .

3o. Umpleby, R. J.; Baxter, S. C.; Rampey, A. M.; Rushton, G. T.; Chen, Y. Z.; Shimizu, K. D., Characterization of the Heterogeneous Binding Site Affinity Distributions in Molecularly Imprinted Polymers. J. Chromatogr. B 2004, 804, 141-149.

31. Dourado, E. M. A.; Herdes, C.; Van Tassel, P. R.; Sarkisov, L., Molecular Recognition Effects in Atomistic Models of Imprinted Polymers. Int. J. Molec. Sci. 2011, 12, 4781-4804. 
SYNOPSIS TOC (Word Style "SN_Synopsis_TOC") . If you are submitting your paper to a journal that requires a synopsis graphic and/or synopsis paragraph, see the Instructions for Authors on the journal's homepage for a description of what needs to be provided and for the size requirements of the artwork.

\section{Insert Table of Contents artwork here}

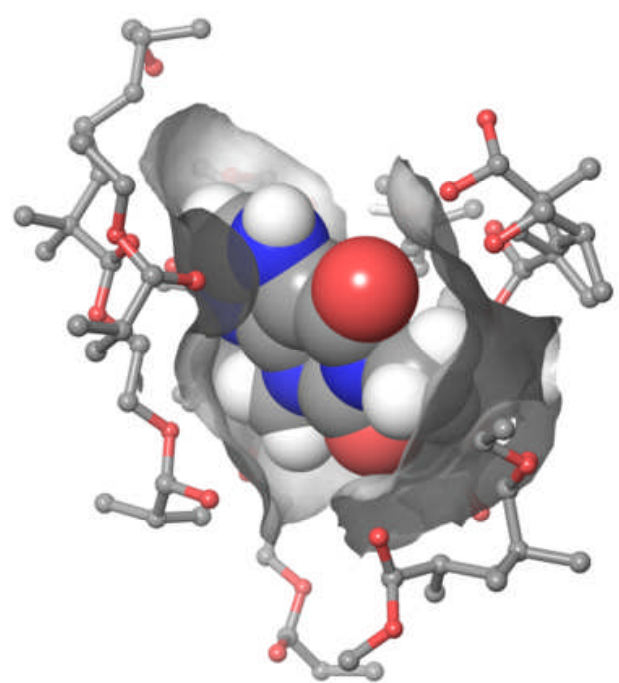

